\title{
Perceived Realism of Pedestrian Crowds Trajectories in VR
}

\author{
Daniele Giunchi* \\ University College London \\ London, UK \\ Fotis Liarokapis \\ CYENS - Centre of Excellence \\ Nicosia, Cyprus
}

\author{
Riccardo Bovo* \\ Imperial College London \\ London, UK \\ Alastair Shipman \\ Imperial College London \\ London, UK
}

\author{
Panayiotis Charalambous \\ CYENS - Centre of Excellence \\ Nicosia, Cyprus \\ Stuart James \\ VGM \& PAVIS, Istituto Italiano di \\ Tecnologia \\ Genova, Italy
}

\author{
Anthony Steed \\ University College London \\ London, UK
}

\author{
Thomas Heinis \\ Imperial College London \\ London, UK
}
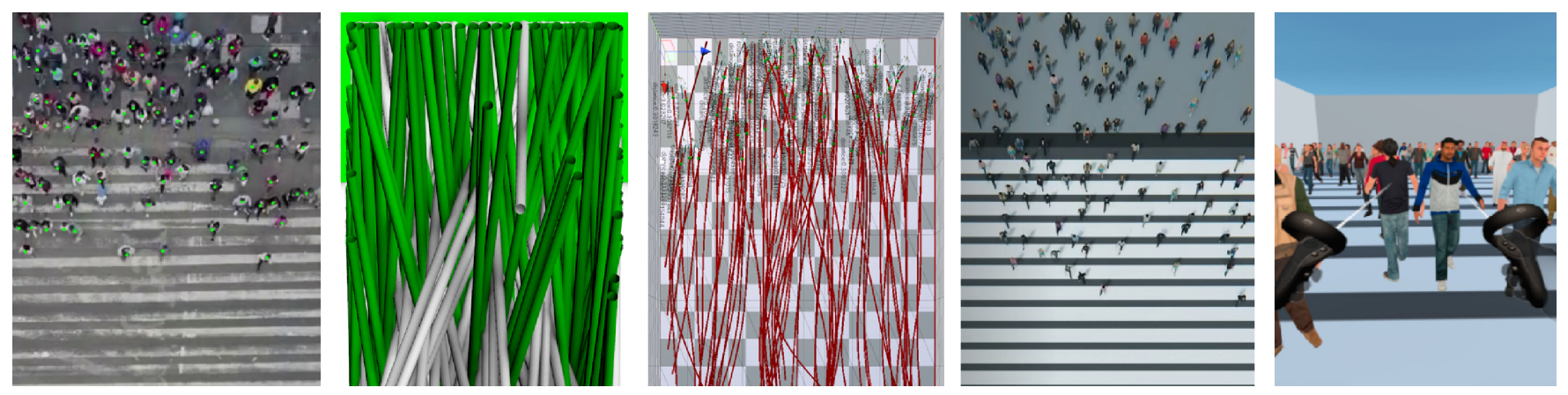

Figure 1: From left to right, real data trajectories, rectilinear trajectory generation, animation process, aerial view, immersive environment.

\begin{abstract}
Crowd simulation algorithms play an essential role in populating Virtual Reality (VR) environments with multiple autonomous humanoid agents. The generation of plausible trajectories can be a significant computational cost for real-time graphics engines, especially in untethered and mobile devices such as portable VR devices. Previous research explores the plausibility and realism of crowd simulations on desktop computers but fails to account the impact it has on immersion. This study explores how the realism of crowd trajectories affects the perceived immersion in VR. We do so by running a psychophysical experiment in which participants rate the realism of real/synthetic trajectories data, showing similar level of perceived realism.
\end{abstract}

${ }^{*}$ Both authors contributed equally to this research.

\footnotetext{
Permission to make digital or hard copies of all or part of this work for personal or classroom use is granted without fee provided that copies are not made or distributed for profit or commercial advantage and that copies bear this notice and the full citation on the first page. Copyrights for components of this work owned by others than the author(s) must be honored. Abstracting with credit is permitted. To copy otherwise, or republish, to post on servers or to redistribute to lists, requires prior specific permission and/or a fee. Request permissions from permissions@acm.org.

VRST '21, December 8-10, 2021, Osaka, Japan

(c) 2021 Copyright held by the owner/author(s). Publication rights licensed to ACM. ACM ISBN 978-1-4503-9092-7/21/12 ..\$15.00

https://doi.org/10.1145/3489849.3489860
}

\section{CCS CONCEPTS}

- Computing methodologies $\rightarrow$ Agent / discrete models; • Human-centered computing $\rightarrow$ User studies.

\section{KEYWORDS}

crowd simulation, perception, virtual reality

\section{ACM Reference Format:}

Daniele Giunchi, Riccardo Bovo, Panayiotis Charalambous, Fotis Liarokapis, Alastair Shipman, Stuart James, Anthony Steed, and Thomas Heinis. 2021. Perceived Realism of Pedestrian Crowds Trajectories in VR. In 27th ACM Symposium on Virtual Reality Software and Technology (VRST '21), December 8-10, 2021, Osaka, Japan. ACM, New York, NY, USA, 5 pages. https://doi. $\operatorname{org} / 10.1145 / 3489849.3489860$

\section{INTRODUCTION}

Crowd simulation is the complex process of simulating collective movement of several individual entities such as virtual humans or animals. It is used in numerous contexts, such as urban or evacuation planning, video games, and cinematography. For realistic, high-density crowd simulations the computation and memory cost is significant and requires the use of high-end desktop computers $[9,22]$ or even in some cases distributed computing [14, 23]. Recently, VR games such as Emergence [1] or Humanity [2] focused user experience on crowded environments. Moreover, VR serious games [7] such as evacuation training gained a lot of interest in the last years. The recent emergence of portable VR devices with 
limited computational resources, such as Oculus Quest and Quest 2, challenge developers as their CPU and GPU capabilities are significantly lower than current desktop-class CPU and GPUs. How does a user perceive a crowd simulation as plausible or realistic? This question is challenging as multiple simulation stages are involved, such as rendering, animation, pedestrian dynamics and, sometimes, the observer's interactions with the crowd such as dynamic avoidance of the observer. Prior studies introduce metrics [12, 19], rely on subjective evaluation by performing user tests $[6,18]$ or even compare against real-world crowd data $[5,10,13]$.To the best of our knowledge there is no study that addresses perceptual realism of crowd simulation trajectories in large and high-density crowds from an immersive perspective. From Nelson [16] we defined low crowd density as 1 agent per square meter, medium density as 1.5 agents per square meter and high density as 2 agents per squared meter. Our paper is motivated by the fact that the computation of realistic trajectories in a densely crowded environment might have high computational cost that could be prohibitive to use on untethered VR devices. Running a cheap algorithm that creates trajectories without affecting their perceived realism allows game designers to address more resources to graphic realism, enhanced animations or even a larger number of virtual characters in the scene. The contribution of our study is two-fold: firstly, we identify and validate through a user study (Experiment 1) this computationally inexpensive method. Validation consists of measuring perceived realism to assess the generation of a plausible set of trajectories for a high-density pedestrian crossing scene. An example of such a scenario is two crossing flows of characters; our experiments show the possibility of generalising such results to this category of scenarios. Secondly, we compare via a VR user test (Experiment 2) the perception of realism between the simulated trajectories and a set of real-world trajectories traced from video footage. This experiment allows us to validate rectilinear trajectories realism when observed via head-tracked stereo displays and explore the impact trajectories' realism has on the immersion levels that users experience in VR.

\section{CONCEPT AND MOTIVATION}

We address the challenge of understanding if computationally inexpensive algorithms can generate plausible or even realistic trajectories in a scenario showing pedestrians following urban constraints such as a road crossing. Previous literature [4] suggests that very simple crowd behaviours, such as ones having rectilinear and single speed trajectories can be perceived as plausible while being computationally inexpensive (the rectilinear algorithm we adapt has been reported to be able to handle a higher number of agents per second as compared to previous methods [3]). Trajectory plausibility is affected by motion illusion and viewpoint. Thus, we aim to clarify if the motion illusion effect can be perceived even in this constrained urban condition where pedestrians are moving in opposite directions. Our baseline consists of real trajectories traced from an aerial video of a pedestrian crossing captured by a drone and containing 280 pedestrians. The video allows us to trace pedestrians trajectories and trace the dimension and specifics of the pedestrian crossing scene. The second dataset is subsequently generated using an adaptation of Barut et al. [3]. We adapted Barut et al. 's real-time rectilinear algorithm to produce trajectories of pedestrians on a

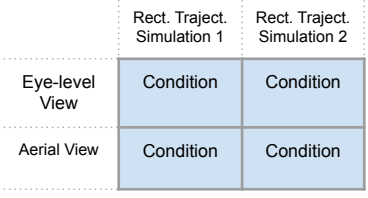

(a) Plausibility Experiment

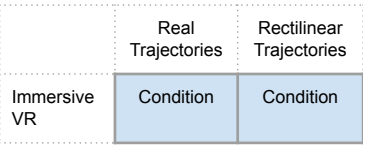

(b) VR Experiment
Figure 2: (a) In the first experiment a total of 4 experimental conditions were defined by the two independent variables of view angle and simulation id; (b) the second experiment only had one independent variable (Real vs Simulated Trajectories) and therefore 2 experimental conditions.

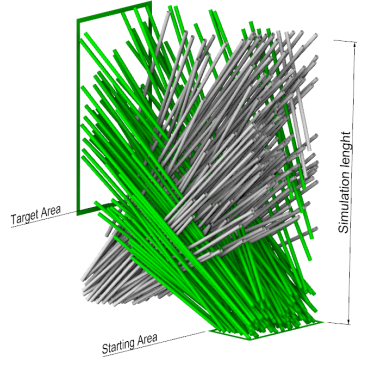

(a) Rectilinear Trajectories

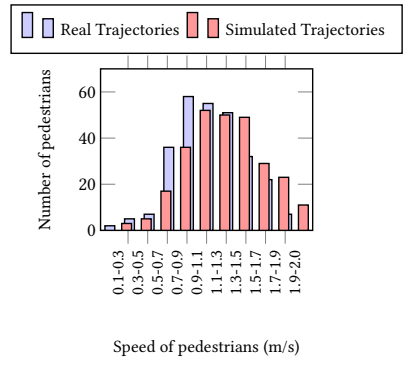

(b) Speed distribution
Figure 3: (a) Adaptation of the rectilinear algorithm proposed by [3], in order to simulate a pedestrian crossing. Our adaptation consisted of adding four constraint areas for the generation of the trajectories and the target location. Each of the pipes represents the trajectory of one pedestrian while the radius represents the clearance between pedestrians; the vertical axis time. (b) The plot shows the average speed distribution of the real data trajectories and the fixed speed distribution of the simulated data trajectories.

crosswalk or pedestrian crossing. The previous literature review surrounding perceptual realism of crowds within psychophysical experiments uses a number of different approaches to measure realism. Some employ simple binary scores (is the crowd realistic or not) [6] whereas others instead ask participants to asses realism using a continuous scale [17]. While the first approach asks participants to make a clear decision, the second allows them to be less deterministic and express their perception with a higher degree of accuracy. Within our questionnaire, we decided to use both approaches. We gather two different levels of perception: a binary classification and a numeric evaluation. Immersion is defined as the "illusion" that "the virtual environment technology replaces the user's sensory stimuli with the virtual sensory stimuli" [21]. The realism of the virtual environment is among those characteristics of the experience that influence the sense of immersion [15]. We use Tcha et al. [21] questionnaire that aimed to measure the experienced immersion levels.

\section{FIRST EXPERIMENT}

The purpose of the first experiment is to validate the plausibility of the rectilinear algorithm proposed by [3] in a realistic scenario. 
Our hypothesis is that rectilinear trajectories in such scenario manifest the same perceptual realism experienced in Barut's work. A within-group experimental design was adopted, where all participants are exposed to all experimental conditions consisting of two different points of view: eye and aerial levels (Fig. 2a). During each experimental session, each participant is exposed to two videos from two different points of view and, after seeing each video, they are asked to fill the questionnaire. We generated two synthetic simulations that were rendered from two different camera angles. In this way, each user was exposed to different simulations when changing point of view, avoiding bias. In both cases, the entry and exit positions alongside the time that pedestrians appeared in the real-world video were used to initialize starting areas, goal areas and preferred velocities for each simulated character. For the preferred velocities in particular, a Monte Carlo approach was used; preferred speeds were sampled from the speed distribution of the actual pedestrians. Running this algorithm twice gives different results though all simulated results generate rectilinear collision free paths that satisfy the speed distribution of the input data. The generated trajectories were then imported into the Unity Game Engine where Rocketbox characters [8] were used to represent each simulated pedestrian. Rocketbox graphical fidelity is typical of VR game characters and crowd simulations without compromising the performances of Oculus Quest, especially when animated. The online test was accessible for 4 weeks and we recruited 153 participants who performed the experiment remotely. Potential participants were contacted via email, and if interested in taking part, they were provided a link to the questionnaire. A randomized sequence of two videos from two viewpoints were generated. The sequence contained both simulations but viewed from different positions. Before viewing each videos participant were instructed to pay particular attention to the trajectory and speed of pedestrians. After viewing each of the videos participants were required to complete the perceived realism questionnaire.

\subsection{Results}

Most participants reported the rectilinear algorithm as either realistic or plausible ( $75 \%$ on the eye-level and $80 \%$ on the aerial view condition).

The large majority of participants, 124, used Desktop computers, 24 used mobile phones, while only 5 tablets. There is no significant difference between overall results and outcomes from participants that used Desktop. The Wilcoxon signed-rank test indicated that perceived realism in the aerial view was statistically significantly higher than in the eye-level view $(Z=2079, p<0.034$, Fig 4a). In addition, the Wilcoxon test indicated that perceived realism for simulation 1 was not statistically significantly different than simulation 2 view $(Z=717.500, p<0.11)$. We further analyzed if the point of view implies any statistical significance related to trajectory linearity perception (manoeuvres) and speed changes, by performing the Wilcoxon signed-rank test again. Trajectory linearity perception in the aerial view was higher than in the eyelevel view $(Z=1247, p<0.001$, Fig 4 b) while Wilcoxon test did not show significant statistical differences in speed homogeneity measures across the point of view $(Z=559, p<0.125$, Fig 4 c).

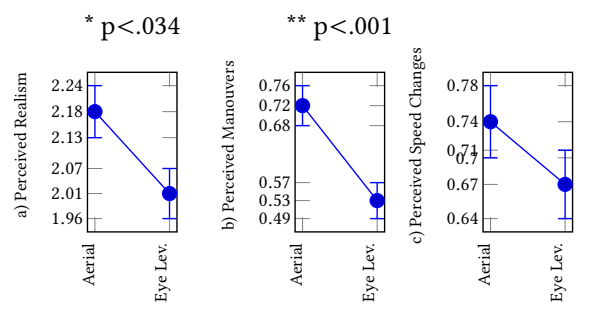

Figure 4: Results from Experiment 1 on the $X$ axes the experimental conditions. a) $Y$ axes: the perceived realism score collected with the realism questionnaire $Q 3$ range between 0 and 3 where 0 is impossible and 3 is realistic. b) Perceived Manoeuvres collected with the realism questionnaire Q1; range between 0 and 1 where 0 represent pedestrian moving in straight trajectories and 1 moving in curved trajectories to avoid collisions. c) Perceived speed changes collected with the realism questionnaire $Q 2$; where 0 represent constant speed and 1 speed changes.

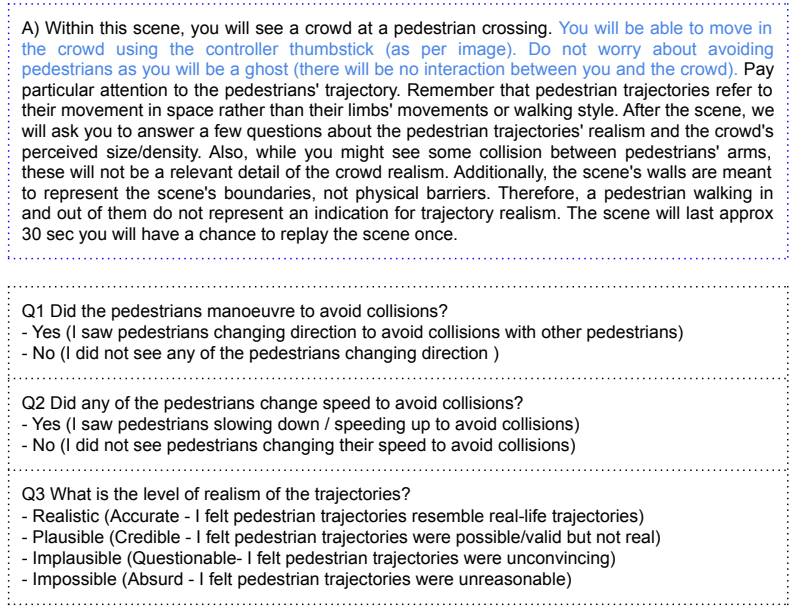

Figure 5: Instructions, for both experiments at the top. Blue text was displayed in experiment 2 . On the bottom Realism questionnaire.

\section{SECOND EXPERIMENT}

This experiment's aims were first to understand if there was a difference in the perceived realism of a crowd visualised with real trajectories compared with synthetic and rectilinear trajectories when the user is immersed in a virtual environment and secondly to measure how trajectories realism affects the level of immersion experienced by users. We asked the participants 3 questions related to the realism perception as in the first experiment: firstly, a direct question about the perceived realism (Fig. 6 a). Secondly, if they noticed manoeuvres to avoid collision by the characters (Fig. 6b) and last if the characters speed changed to avoid collision (Fig. 6c). We adopted a within-group experimental design in which all participants are exposed to both of the realism conditions (i.e. real data and simulated data) as shown in Fig. 2b. The video length is inherited from real data, to compare the simulated data fairly with real data we had to generate a simulation of equal duration. To counterbalance any possible ordering effects, the sequence of conditions is randomized for each participant. Before each experiment, 
we collected consent forms and demographics. Across every trial, we collected the realism questionnaire. The real-world trajectories were tracked from a 28 seconds video bought and downloaded from iStock ${ }^{1}$ using the semi-automatic procedure of tracking heads The synthetic data were generated using the linear trajectories algorithm.We recruited 40 participants, 15 participants performed the VR experiment remotely, while the remaining 25 took part in the experiment in a controlled environment. The application was pre-installed on an Oculus Quest 2 for the controlled experiment participants. Once the application was downloaded/installed and opened, a series of graphical instructions informed the participant about the aim of the project as well as asking for the consent of the participant, which was recorded, then demographic were collected. After the experiment started, randomly selecting which experiment to be shown first, instructions were shown explaining to the participant that they would be asked to observe a crowd and subsequently asked to rate the realism of the crowd trajectories. The participants were not aware about the nature of the crowd data (simulated or real). Participants were asked to focus on the trajectories rather than the humanoid movements or the environment details.The scene was created in Unity and walls were placed on the borders to focus the participants on the region of interest. Between each trial, participants were asked to fill the trajectories' realism questionnaire. (Fig. 5). The user could perform the session seated or standing, and could move freely in the digital environment with Oculus controllers, similarly to movemement in a game scene. To simulate a typical game scenario, the users were not allowed to stop the simulation to investigate the scene. To prevent indecision from the participants, we gave the possibility to repeat the session once, and set a scale in the questionnaire's answer. Users were informed of this possibility before starting the experiment; we limited the repetition capability to one repetition to avoid unbalanced sessions between participants, and to limit experiment time.
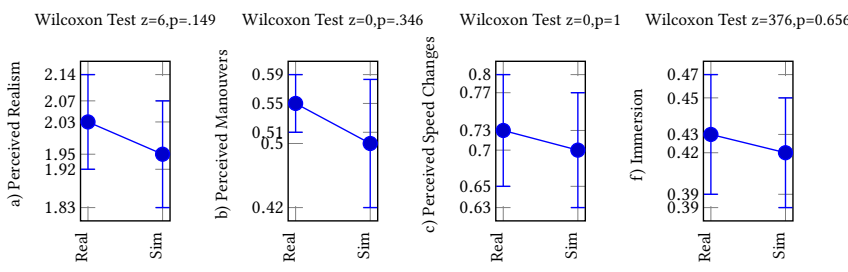

Figure 6: On the $X$ axis experimental conditions: Real and Simulated trajectories, the error bars represent Standard Error. a) $Y$ axes: perceived realism ( 0 is impossible and 3 is realistic). b) $Y$ axes: perceived manoeuvres range 0 to 1 (0 trajectories perceived as straight and 1 pedestrian perceived as maneuvering) c) $\mathrm{Y}$ axes: perceived speed changes between 0 and 1 (0 pedestrian moving at a fix speed and 1 pedestrian changing speed). f) $Y$ axes: Reported level of immersion (0 low immersion and 1 high immersion).

The VRCrowd application is developed with Unity 2019.4.0 and uses Google Firebase to collect data. A network connection needs to be present to run the experiment. We showed two sets of trajectories: real trajectories traced from the aerial video, and synthetic

\footnotetext{
${ }^{1}$ https://www.istockphoto.com/it/video/veduta-aerea-di-una-traversata-a-cittá-delmessico-gm1018488334-273794051
}

trajectories. To increase model animation realism and model visual appearance, we animated Rocketbox characters [8] using the phase functional neural networks animation system from Holden $e t$ al. [11]. For both real and artificial trajectories, we applied the offline animations by processing the trajectories in Unity. Then we stored the dataset in binary files to be played within the Oculus.

\subsection{Results}

We did not measure significant differences between real and synthetic data from both the answers related to collision avoidance and speed changes. While participants were undecided if the pedestrians changed trajectories to avoid collisions, $70 \%$ of the participants reported no speed changes. Perception of manoeuvring in the real data condition was not significantly different $(Z=0, p<.346$, Fig. $6 b)$ across the real/simulated data. This was also the case for perceived speed changes $(Z=0, p=1$, Fig. $6 \mathrm{c})$. Such similarity between perceptions of simulated and real data suggests that when the user is immersed, rectilinear trajectories are perceived as similarly realistic as real trajectories.Similar results were also visible in the answers distribution for the perceived realism, and also between the controlled users and the group that performed remotely. The Wilcoxon signed-rank test indicated that the perceived realism of real data was not statistically different than simulated data $(\mathrm{Z}=6, \mathrm{p}<.149$, Fig. $6 \mathrm{a})$. We aim to understand if the trajectory realism affects the immersion level. We compare the scores distributions of the immersion questionnaire [21]. The Wilcoxon signed-rank test indicates that immersion level reported by participants across the two levels is not statistically significantly different $(Z=376, p<.656$, Fig. 6f).

\section{FUTURE WORKS \& CONCLUSION}

Despite our scenario's specificity, this study aims to investigate a common configuration when crowd simulations are evaluated. We aim to extend the study by increasing the number of different situations such as singular flow, four flows in a crossroad context, and bottleneck scenarios. Our experiment exposes the participants to visual stimuli originated by crowd simulations. A suggestive follow up can introduce auditory cues as studied by Stanton et al. [20], analysing perception and immersion. First experiment outcomes illustrates how the adapted algorithms from [3] can be used to generate plausible/realistic trajectories in conditions in which the flow of pedestrians is not random as in the case of a pedestrian crossing. The results from the second experiment underlines how, in an immersive condition, we can not determine differences in perceived realism between traced trajectories and simulated trajectories. The results highlight how the different levels of immersion experienced by users are not statistically different across the conditions of real and simulated trajectories. This outcome suggests that spending computational power for trajectory realism does not increase the perceived realism in a high density crowd crosswalk setting.

\section{ACKNOWLEDGMENTS}

This research has been supported by the H2020 EU project under grant agreement No 739578 (RISE - Call: H2020-WIDESPREAD-012016-2017-TeamingPhase2) and the Government of the Republic of Cyprus through the Directorate General for European Programmes, Coordination and Development. 


\section{REFERENCES}

[1] [n.d.]. Emergence. https://store.steampowered.com/app/1337820/Emergence/

[2] [n.d.]. Humanity. https://uploadvr.com/psvr-humanity-2021/

[3] Oner Barut and Murat Haciomeroglu. 2015. Real-time collision-free linear trajectory generation on GPU for crowd simulations. Visual Computer 31, 6-8 (2015), 843-852. https://doi.org/10.1007/s00371-015-1105-z

[4] Oner Barut, Murat Haciomeroglu, and Ebru Akcapinarsezer. 2018. Perceptual evaluation of maneuvering motion illusion for virtual pedestrians. Visual Computer 34, 6-8 (6 2018), 1119-1128. https://doi.org/10.1007/s00371-018-1557-z

[5] Panayiotis Charalambous, Ioannis Karamouzas, Stephen J. Guy, and Yiorgos Chrysanthou. 2014. A Data-Driven Framework for Visual Crowd Analysis. Computer Graphics Forum 33, 7 (Oct. 2014), 41-50. https://doi.org/10.1111/cgf.12472

[6] Cathy Ennis, Christopher Peters, and Carol O'sullivan. 2011. Perceptual effects of scene context and viewpoint for virtual pedestrian crowds. ACM Transactions on Applied Perception 8, 2 (1 2011), 1-22. https://doi.org/10.1145/1870076.1870078

[7] Zhenan Feng, Vicente A González, Robert Amor, Ruggiero Lovreglio, and Guillermo Cabrera-Guerrero. 2018. Immersive virtual reality serious games for evacuation training and research: A systematic literature review. Computers \& Education 127 (2018), 252-266.

[8] Mar Gonzalez-Franco, Eyal Ofek, Ye Pan, Angus Antley, Anthony Steed, Bernhard Spanlang, Antonella Maselli, Domna Banakou, Nuria Pelechano, Sergio OrtsEscolano, Veronica Orvalho, Laura Trutoiu, Markus Wojcik, Maria V. SanchezVives, Jeremy Bailenson, Mel Slater, and Jaron Lanier. 2020. The Rocketbox Library and the Utility of Freely Available Rigged Avatars. Frontiers in Virtual Reality 1, November (2020), 1-23. https://doi.org/10.3389/frvir.2020.561558

[9] Stephen J. Guy, Jatin Chhugani, Sean Curtis, Pradeep Dubey, Ming C. Lin, and Dinesh Manocha. 2010. PLEdestrians: A Least-Effort Approach to Crowd Simulation. In Proceedings of the 2010 Eurographics/ACM SIGGRAPH Symposium on Computer Animation, SCA 2010, Madrid, Spain, 2010, Zoran Popovic and Miguel A Otaduy (Eds.). Eurographics Association, 119-128. https://doi.org/10.2312/SCA/ SCA10/119-128

[10] Stephen J. Guy, Jur Van Den Berg, Wenxi Liu, Rynson Lau, Ming C. Lin, and Dinesh Manocha. 2012. A statistical similarity measure for aggregate crowd dynamics. In ACM Transactions on Graphics, Vol. 31. 1-11. https://doi.org/10. 1145/2366145.2366209

[11] Daniel Holden, Taku Komura, and Jun Saito. 2017. Phase-functioned neural networks for character control. ACM Transactions on Graphics 36, 4 (2017), 1-13. https://doi.org/10.1145/3072959.3073663

[12] Mubbasir Kapadia, Shawn Singh, Brian Allen, Glenn Reinman, and Petros Falout sos. 2009. SteerBug: an interactive framework for specifying and detecting steering behaviors. In Proceedings of the 2009 ACM SIGGRAPH/Eurographics Symposium on Computer Animation - SCA '09. ACM Press, New Orleans, Louisiana, 209. https://doi.org/10.1145/1599470.1599497

[13] Alon Lerner, Yiorgos Chrysanthou, Ariel Shamir, and Daniel Cohen-Or. 2010. Context-Dependent Crowd Evaluation. Computer Graphics Forum 29, 7 (Sept. 2010), 2197-2206. https://doi.org/10.1111/j.1467-8659.2010.01808.x

[14] Artur Malinowski, Paweł Czarnul, Krzysztof Czuryło, Maciej Maciejewski, and Paweł Skowron. 2017. Multi-agent large-scale parallel crowd simulation. Procedia Computer Science 108 (Jan. 2017), 917-926. https://doi.org/10.1016/j.procs.2017. 05.036

[15] Rory McGloin, Kirstie M. Farrar, and Marina Krcmar. 2011. The impact of controller naturalness on spatial presence, gamer enjoyment, and perceived realism in a tennis simulation video game. Presence: Teleoperators and Virtual Environments 20, 4 (2011), 309-324. https://doi.org/10.1162/PRES\{_\}a\{_\}00053

[16] Michael G. Nelson, Alexandros Koilias, Sahana Gubbi, and Christos Mousas. 2019 Within a virtual crowd: Exploring human movement behavior during immersive virtual crowd interaction. In Proceedings - VRCAI 2019: 17th ACM SIGGRAPH International Conference on Virtual-Reality Continuum and its Applications in Industry. Association for Computing Machinery, Inc, New York, NY, USA, 1-10. https://doi.org/10.1145/3359997.3365709

[17] Stuart O'Connor, Fotis Liarokapis, and Christopher Peters. 2013. An initial study to assess the perceived realism of agent crowd behaviour in a virtual city. In 2013 5th International Conference on Games and Virtual Worlds for Serious Applications, VS-GAMES 2013. https://doi.org/10.1109/VS-GAMES.2013.6624220

[18] Nuria Pelechano, Catherine Stocker, Jan Allbeck, and Norman Badler. 2008. Being a part of the crowd: Towards validating VR crowds using presence. Proceedings of the International foint Conference on Autonomous Agents and Multiagent Systems, AAMAS 1, May (2008), 135-141. https://doi.org/10.1145/1402383.1402407

[19] Shawn Singh, Mubbasir Kapadia, Petros Faloutsos, and Glenn Reinman. 2009 SteerBench: a benchmark suite for evaluating steering behaviors. Computer Animation and Virtual Worlds 20, 5-6 (Sept. 2009), 533-548. https://doi.org/10. 1002/cav.277

[20] Tasha R. Stanton and Charles Spence. 2020. The Influence of Auditory Cues on Bodily and Movement Perception. Frontiers in Psychology 10 (2020), 3001. https://doi.org/10.3389/fpsyg.2019.03001
[21] Katy Tcha-Tokey, Olivier Christmann, Emilie Loup-Escande, and Simon Richir. 2016. Proposition and Validation of a Questionnaire to Measure the User Experience in Immersive Virtual Environments. International fournal of Virtual Reality 16, 1 (2016), 33-48. https://doi.org/10.20870/ijvr.2016.16.1.2880

[22] Wouter van Toll, Atlas F. Cook IV, and Roland Geraerts. 2012. Real-time densitybased crowd simulation. Comput. Animat. Virtual Worlds 23, 1 (2012), 59-69. https://doi.org/10.1002/cav.1424

[23] Guillermo Vigueras, Juan M. Orduña, Miguel Lozano, and Yiorgos Chrysanthou. 2011. A distributed visualization system for crowd simulations. Integrated Computer-Aided Engineering 18, 4 (Jan. 2011), 349-363. https://doi.org/10.3233/ ICA-2011-0381 Publisher: IOS Press. 